Vacuum ultraviolet excitation luminescence spectroscopy of few-layered $\mathrm{MoS}_{2}$

This content has been downloaded from IOPscience. Please scroll down to see the full text.

2016 J. Phys.: Condens. Matter 28015301

(http://iopscience.iop.org/0953-8984/28/1/015301)

View the table of contents for this issue, or go to the journal homepage for more

Download details:

IP Address: 128.179.254.126

This content was downloaded on 18/12/2015 at 23:04

Please note that terms and conditions apply. 


\title{
Vacuum ultraviolet excitation luminescence spectroscopy of few-layered $\mathrm{MoS}_{2}$
}

\author{
V Pankratov ${ }^{1,5}$, J Hoszowska ${ }^{2,5}, \mathrm{~J}-\mathrm{Cl}$ Dousse $^{2}$, M Huttula ${ }^{1}$, A Kis $^{3}$, \\ D Krasnozhon ${ }^{3}$, M Zhang ${ }^{4}$ and W Cao ${ }^{1}$
}

${ }^{1}$ Research Centre for Molecular Materials, PO Box 3000, FIN- 90014, University of Oulu, Finland

2 Department of Physics, University of Fribourg, Ch. du Musée 3, CH-1700 Fribourg, Switzerland

3 Electrical Engineering Institute, École Polytechnique Fédérale de Lausanne (EPFL),

CH-1015 Lausanne, Switzerland

${ }^{4}$ Department of Physics, East China University of Science and Technology, Shanghai 200237, People's

Republic of China

E-mail: mzhang@ecust.edu.cn and wei.cao@oulu.fi

Received 10 September 2015, revised 6 November 2015

Accepted for publication 16 November 2015

Published 9 December 2015

\begin{abstract}
We report on vacuum ultraviolet (VUV) excited photoluminescence (PL) spectra emitted from a chemical vapor deposited $\mathrm{MoS}_{2}$ few-layered film. The excitation spectrum was recorded by monitoring intensities of PL spectra at $\sim 1.9 \mathrm{eV}$. A strong wide excitation band peaking at $7 \mathrm{eV}$ was found in the excitation. The PL excitation band is most intensive at liquid helium temperature and completely quenched at $100 \mathrm{~K}$. Through first-principles calculations of photoabsorption in $\mathrm{MoS}_{2}$, the excitation was explicated and attributed to transitions of electrons from $\mathrm{p}$ - and d-type states in the valence band to the d-and p-type states in the conduction band. The obtained photon-in/photon-out results clarify the excitation and emission behavior of the low dimensional $\mathrm{MoS}_{2}$ when interacting with the VUV light sources.
\end{abstract}

Keywords: low dimensional $\mathrm{MoS}_{2}$, excitation spectrum, first-principles calculations, photoluminescence

(Some figures may appear in colour only in the online journal)

\section{Introduction}

The past decade has witnessed a boost of the layered crystals researches since the pioneering (re)discovery of the monolayer graphene $[1,2]$ and enormous applications based on these materials [3]. Inorganic layered crystals (ILCs), similar to their organic counterparts, are formed through covalent bonds in layers and van der Waals forces between layers. Benefiting from intra- and inter- layer structures, it is relatively easy to manipulate complex structures to reach different application purposes. In contrast to the gapless pristine graphene, most of the ILCs have non-zero bandgaps. Such semiconducting properties are essential in industrial applications.

${ }^{5}$ Authors who contributed equally to this work.
The library of ILCs consists of various species of nitrides, and metal compounds of the group VI [4]. Among them, the $\mathrm{MoS}_{2}$ is well-known as a conventional lubricant [5] and catalyst for hydrogen evolution $[6,7]$. Recent investigations of the materials showed that mono- and few- layered $\mathrm{MoS}_{2}$ are also advanced in high efficient transistors [8,9], photoelectronics [10], and electrocatalysis [11]. To improve the properties of the $\mathrm{MoS}_{2}$-based devices, developments of molecular modulation and engineering are in progress. Different methods have been applied to synthesize $\mathrm{MoS}_{2}$ monolayers [12, 13], and few layers on a large scale $[14,15]$.

Exploration of the $\mathrm{MoS}_{2}$ semiconductor functionalities was featured from a discovery of the transformation of indirect to direct band transitions when lowering the ILC dimensions $[16,17]$. Laser-induced photoluminescence (PL) spectra 
showed that a direct band transition peaking at an energy of $\sim 1.9 \mathrm{eV}$ in a single layer diminished with the increase of film thickness. Such a PL peak was also found in the few layered $\mathrm{MoS}_{2}$ at low temperatures but the intensity decreased when temperature increased [18]. The temperature dependent PL spectroscopy of mono-layer $\mathrm{MoS}_{2}$ shows a low-energy peak beside the dominating one due to a bound exciton state [19]. Relaxing dynamics and time scales for the different relaxation processes were also elucidated [14], while studies of the excitons were further extended to other low dimensional metal dichalcogenides [20]. Extensive studies through this PL technique further revealed that indirect band transitions were plasmonic enhanced in nanoparticles [21], and also Au nanoparticle decorated flakes [22].

Despite the pioneering findings revealed via laser-induced PL detections, the laser-induced spectroscopies, carried out with blue lasers as incident sources are limited in photon energy variations to excite valence band (VB) electrons to higher conduction bands (CBs) or electrons from deeper levels to the CBs. A detailed knowledge of the electronic structures is of crucial importance for digging out materials' potentials in different applications. Though VB structures of the cleaved $\mathrm{MoS}_{2}$ single-crystal flake were revealed by the angularresolved photoemission spectroscopy (ARPES) [23], the probe of the $\mathrm{CB}$ structure is beyond the reach of the ARPES technique. The CBs were also investigated through the x-ray absorption spectroscopy, but so far these studies were applied only to the bulk $2 \mathrm{H}-\mathrm{MoS}_{2}[24,25]$. More band structures and transition mechanisms of the low-dimensional $\mathrm{MoS}_{2}$ await to be revealed, however, with incident sources of tunable photon energies. In this paper, we report a photoluminescence study of few-layered $\mathrm{MoS}_{2}$ excited by energy tunable synchrotron radiation. By monitoring the PL emission and tuning the SR beam in the VUV energy region, a wide excitation band peaking at $7 \mathrm{eV}$ was found. The excitation band was strong at low temperature but disappeared at temperature above $100 \mathrm{~K}$. First-principles calculations were carried out to explicate the origin of the excitation band.

\section{Experimental}

Large-area few-layer $\mathrm{MoS}_{2}$ was grown using the two-step thermolysis process [26]. High-purity $\left(\mathrm{NH}_{4}\right)_{2} \mathrm{MoS}_{4}$ was added to $20 \mathrm{ml}$ of dimethylformamide (DMF) to form a $1.25 \mathrm{wt} \%$ solution. The solution was sonicated for $20 \mathrm{~min}$ before use. Sapphire substrates were cleaned with piranha solution $\left(\mathrm{H}_{2} \mathrm{SO}_{4} / \mathrm{H}_{2} \mathrm{O}_{2}\right)$. We used highly polished, EPI-ready grade sapphire substrates. In order to achieve atomically smooth sapphire terraces on the surface we annealed the substrates in air for $1 \mathrm{~h}$ at a temperature of $1150{ }^{\circ} \mathrm{C}$ just prior to the growth process. The next step of the growth was the immersion of the substrates into the $\left(\mathrm{NH}_{4}\right)_{2} \mathrm{MoS}_{4}$ solution. The substrates were then baked on a hot plate at $120^{\circ} \mathrm{C}$ for $30 \mathrm{~min}$.

The prepared samples with the fresh thin film of $\left(\mathrm{NH}_{4}\right)_{2} \mathrm{MoS}_{4}$ were placed in the cold zone of the quartz tube with a gas mixture $\left(\mathrm{Ar} / \mathrm{H}_{2} 20 / 5 \mathrm{sccm}\right)$. When the center of the furnace reached $500{ }^{\circ} \mathrm{C}$, we moved the crucible with the samples inside the tube by using a magnet. After $1 \mathrm{~h}$, the crucible was moved to the cold zone and the gas environment was changed to Ar. The second step of the growth consists in sulfurization of the film in the environment of Ar and sulfur at $1000^{\circ} \mathrm{C}$. The sulfur is used as a protection gas against the oxidation. The sulfur is introduced into the chamber in a pellets form which becomes sulfur vapor at the growth temperature. Samples were further characterized through optical microscopy and atomic force spectroscopy (AFM).

VUV excitation luminescence spectroscopy is especially a powerful tool for investigating wide band gap materials such as fluorides [27], iodides [28] and oxides [29-32]. The method was extended to studies of electronic excitations in semiconductor nanocrystals [33]. In this work, luminescence properties of $\mathrm{MoS}_{2}$ were investigated in a wide spectral range using synchrotron radiation from the Max III storage ring of the Max IV synchrotron (Lund, Sweden). The photoluminescence endstation was installed on the FinEst branch of the I3 undulator beamline. A $6.65 \mathrm{~m}$ off-axis eagle type normal incidence monochromator was employed, delivering monochromatic photons with a flux of $10^{11}-10^{13} \mathrm{ph} \mathrm{s}^{-1} / 0.1 \% \mathrm{BW}[34,35]$. The incident beam was cut to a size of $\sim 1 \mathrm{~mm} \times 1 \mathrm{~mm}$. The excitation spectra were recorded in the $250-100 \mathrm{~nm}(5-12 \mathrm{eV})$ spectral range with a spectral resolution of $0.3 \mathrm{~nm}$. Excitation spectra of sodium salicylate were employed to normalize the intensity of the photons impinging onto the sample. Luminescence spectra in the visible/infrared range were recorded with a monochromator (SpectraPro-308i, Acton Research Corporation) equipped with a photomultiplier (HAMAMATSU R6358P). Step of the analyzing monochromator was typically $8 \mathrm{~nm}$. Emission spectra were corrected for the spectral response of the detection system. Samples were installed onto the sample holder of a flow-type liquid helium cryostat, allowing temperature changes from 10 to $300 \mathrm{~K}$. Two different beam polarizations (vertical and horizontal) were used. However, emission and excitation spectra were found to be independent of the beam polarization.

\section{Theory}

To calculate the spectroscopic properties of few-layered $\mathrm{MoS}_{2}$, we employed the hybrid exchange-correlation functional Heyd-Scuseria-Ernzerhof (HSE06) [36, 37] by using norm-conserving pseudopotentials implemented in CASTEP code [38]. Calculations were performed at the temperature of $0 \mathrm{~K}$. A vacuum region of $25 \AA$ was applied in the $z$-direction to exclude mirror interactions between neighboring images. The convergence tolerance of the energy was set to $10^{-6} \mathrm{eV}$ and the energy cutoff of the plane wave basis set to $650 \mathrm{eV}$. The Brillouin zone was represented by a set of $10 \times 10 \times 1$ $k$-points for the geometry optimizations, and $15 \times 15 \times 1$ $k$-points were used to obtain the optical properties. Taking the sample thickness into consideration, a bilayer model was employed. A $5 \times 5$ supercell was constructed from 150 atoms including $50 \mathrm{Mo}$ atoms and $100 \mathrm{~S}$ atoms. Interlayer effect of van der Waals interactions was introduced explicitly through the empirical correction scheme proposed by Ortmann, Bechstedt, and Schmidt [39]. 

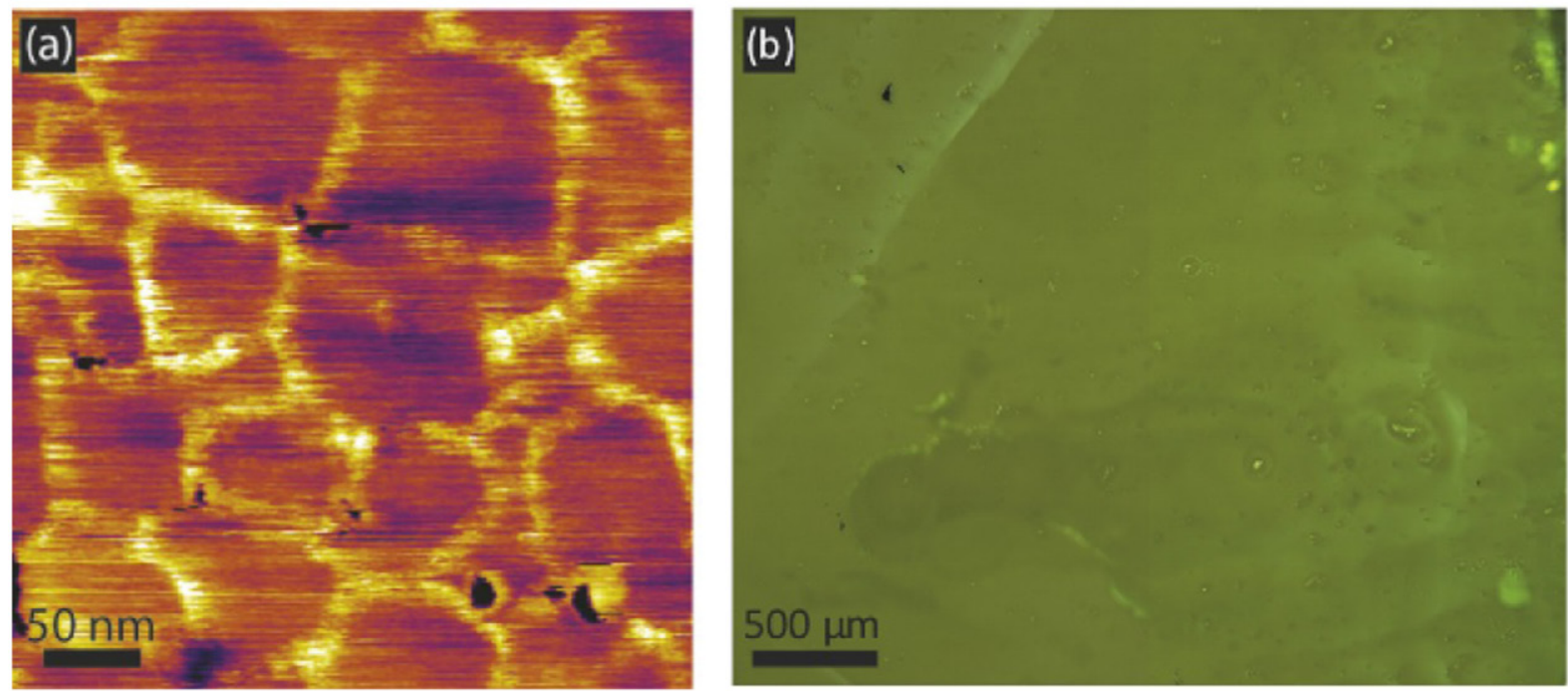

Figure 1. Morphology of the CVD few-layered $\mathrm{MoS}_{2}$ sample probed via (a) the atomic force microscopy, and (b) the optical microscopy.

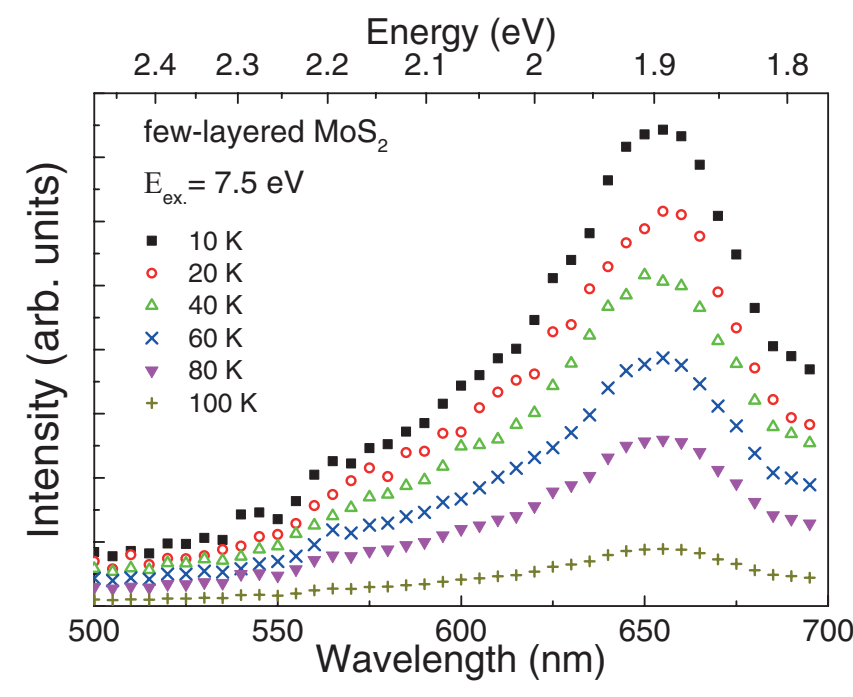

Figure 2. Temperature dependence of photoluminescence spectra of few-layered $\mathrm{MoS}_{2}$ under VUV excitation at the incident photon energy of $E_{\mathrm{ex}}=7.5 \mathrm{eV}$.

\section{Results and discussions}

An AFM image of the sample is shown in figure 1(a). The AFM phase image of the film was acquired directly on top of the sapphire substrate and the AFM was operated in a tapping mode. With the AFM, grains sizes ranging from 100 to $200 \mathrm{~nm}$ can be observed. Crystalline domains boundaries are visible because they are segregated by impurities. This is consistent with the experimental evidence that grain boundaries appear as strong variations in the phase contrast during the AFM scan. Figure 1(b) shows an optical microscopic image of the sample, where light contrast denotes the general flat surface.

The PL spectra of $\mathrm{MoS}_{2}$ sample under VUV excitation in temperature range from 10 to $100 \mathrm{~K}$ are shown in figure 2 . The spectral position and shape of the emission spectra remain the same despite the temperature changes. Each emission spectrum contains a well-resolved emission band peaking at $\sim 1.9 \mathrm{eV}$ and a shoulder at higher energy. Such PL bands

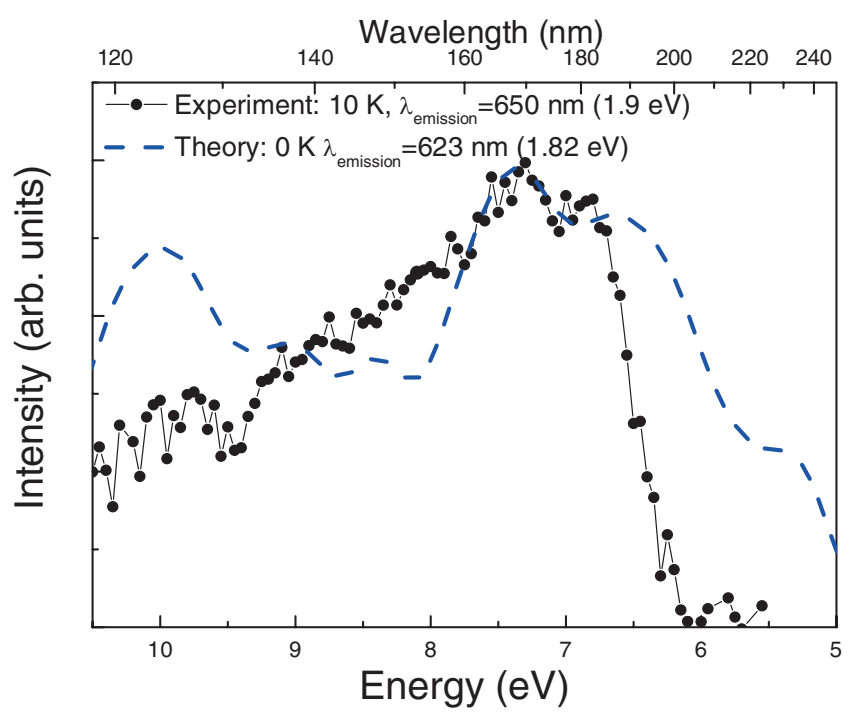

Figure 3. PL excitation spectrum of few-layered $\mathrm{MoS}_{2}$ measured at the temperature of $10 \mathrm{~K}$. The dashed curve represents the theoretical calculation of the excitation spectrum at $0 \mathrm{~K}$.

were reported in previous studies under visible laser excitation [17, 40-42] and attributed to several types of excitons in mono- and few-layered $\mathrm{MoS}_{2}[41,42]$. According to [41, 42] a neutral free A-exciton $\left(\mathrm{X}^{\mathrm{O}, \mathrm{A}}\right)$ is responsible for emission at $1.89 \mathrm{eV}$, while a broad contribution near $2.01 \mathrm{eV}$ stems from $\mathrm{B}$-exciton emission $\left(\mathrm{X}^{\mathrm{O}, \mathrm{B}}\right)$ from the spin-orbit split valence band at the K-points of the Brillouin zone. The emission bands of the negatively charged trion $\left(\mathrm{X}^{\mathrm{T}}\right)$ and a defect induced feature $\left(\mathrm{X}^{\mathrm{D}}\right)$ known in $2 \mathrm{D} \mathrm{MoS}_{2}[42,43]$ have spectral positions below $1.8 \mathrm{eV}$ and could not be detected in figure 2 due to the limitation of the detection system of the set-up.

Figure 2 demonstrates that the PL intensity decreases with increasing temperature. Generally speaking, the PL intensity degradation at high temperatures is typically attributed to the enhancement of the nonradiative electron-hole recombination process which reduces the probability of radiative transitions. In [18] it was demonstrated that the PL intensity of $\mathrm{MoS}_{2}$ under visible laser excitation decreases when the temperature 

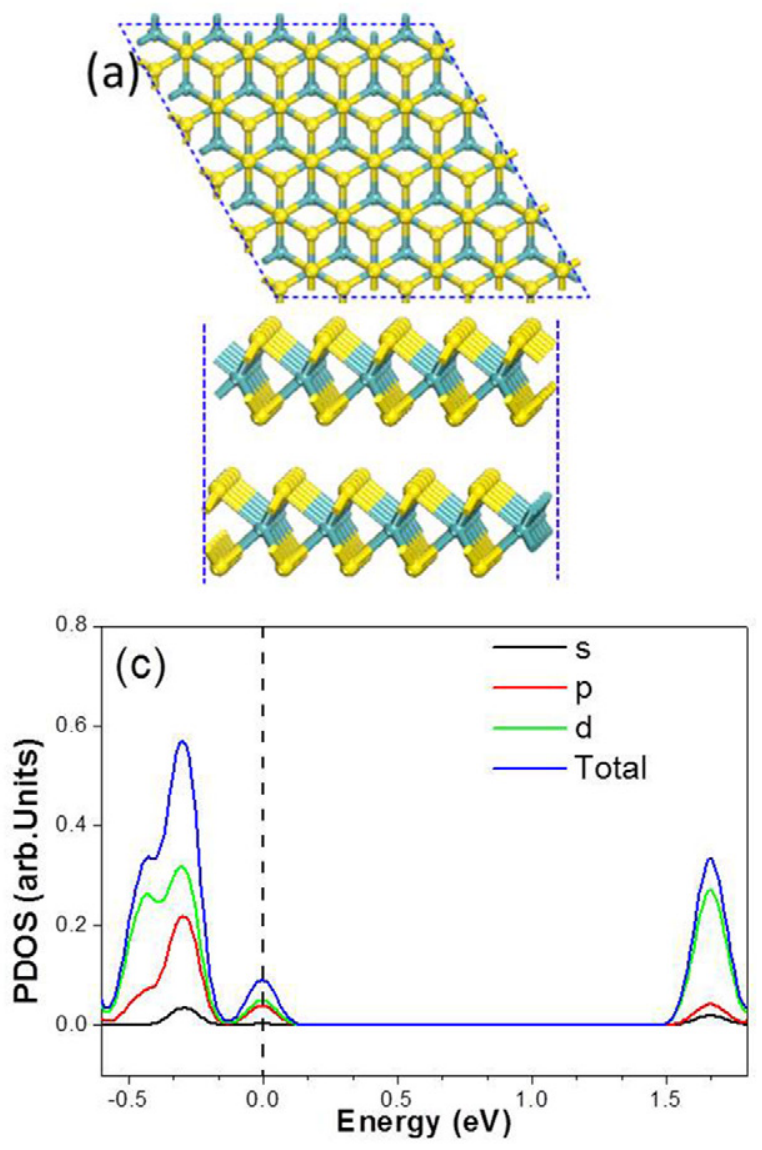
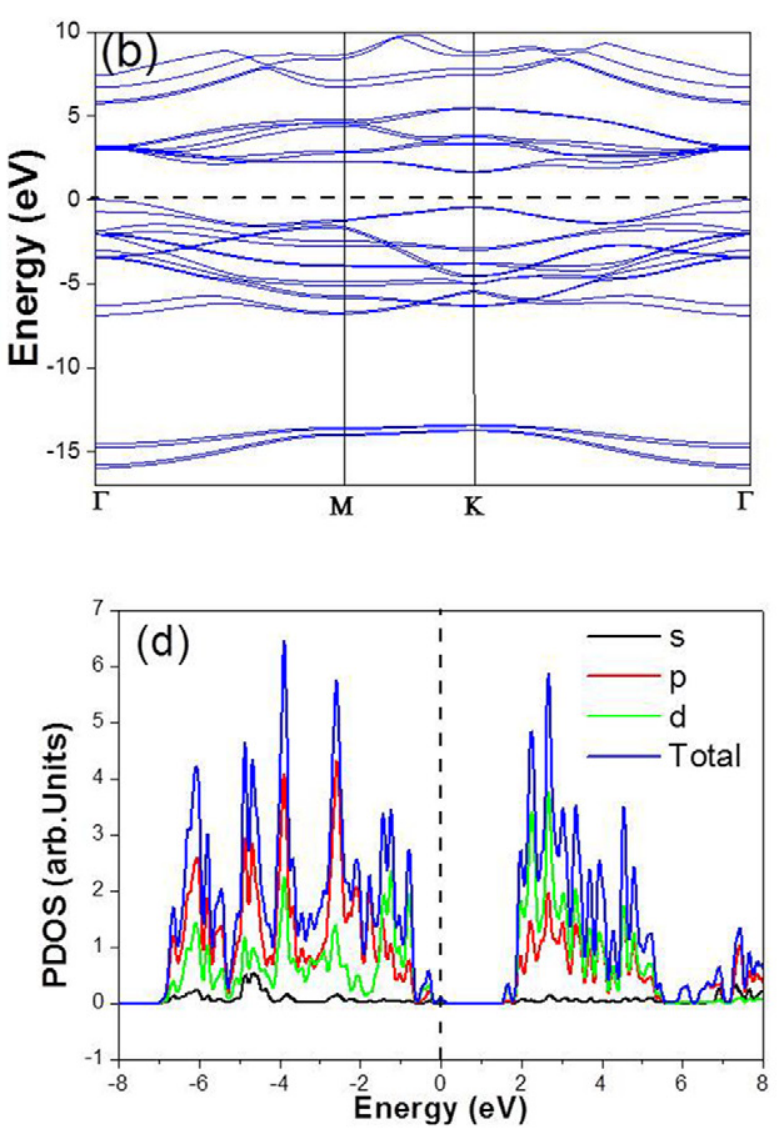

Figure 4. Theoretical calculations of the bilayer $\mathrm{MoS}_{2}$ geometry and electronic structures. In (a), optimized geometric structures of the two-layered $\mathrm{MoS}_{2}$ are shown from the top view and the side view. The blue and yellow spheres represent Mo and S, respectively. The electron band structure of the bilayer is plotted in (b). The partial density of states next to the Fermi level is depicted in (c) and extended in (d). The Fermi level is denoted with dashed lines.

increases from 90 to $423 \mathrm{~K}$ regardless of the layer thickness. It was concluded there that the temperature dependence of the PL intensity in $2 \mathrm{D} \mathrm{MoS}_{2}$ is typical for conventional semiconductors. Therefore, the temperature dependence of the PL signal depicted in figure 2 is in principle in line with the data obtained before using visible excitation.

The effective VUV excited PL spectra at low temperature allowed us to employ higher-energy photons to investigate the excitation spectra of the A-exciton and to get information about intrinsic electronic properties in layered $\mathrm{MoS}_{2}$. Taking into account that intensity of the PL signal is strongest at low temperature, we applied low temperature in order to get intensive and reliable excitation spectra. Figure 3 demonstrates the excitation spectrum by monitoring intensities of the emitted PL around $1.9 \mathrm{eV}$ at a low temperature of $10 \mathrm{~K}$. This spectrum comprises a strong PL excitation band peaking at about $7 \mathrm{eV}$ and having a prolonged shoulder to higher energies. It is important that the PL intensity is low if the energy of excitation is lying in the $5.0-6.5 \mathrm{eV}$ range. This energy range is much higher than the band gap of $\mathrm{MoS}_{2}$ and definitely should be sufficient for excitation of PL. It is worth mentioning that the CB structures were investigated for the bulk $\mathrm{MoS}_{2}$. The s- or d-like DOS near to the sulfur atoms were revealed via soft x-ray absorption near-edge structures (XANES) [24, 25]. However, no general information about the other band transitions from VB to CB could be given by the XANES due to the dominant dipole transition in the core-level excitations of the sulfur $2 p$ electrons [25]. On another hand, to the best of our knowledge, studies of the excitation spectra of the lowdimensional $\mathrm{MoS}_{2}$ are still lacking.

Combining the theoretical and experimental results allows us to clarify the emission and excitation schemes. The bilayer $\mathrm{MoS}_{2}$ was chosen here, considering the rather huge synchrotron beam coverage area on the CVD sample. The theoretical excitation spectrum of the bilayer is depicted in figure 3 to make a direct comparison with the experimental one. The optimized geometry is shown in figure 4(a) for the top and side views. The lattice parameter of the two-layered $\mathrm{MoS}_{2}$ from our calculations is $3.21 \AA$. The band structure is shown in figure 4(b), the projections of the density of states (DOS) next to the Fermi level in figure 4(c), and the DOS in an enlarged energy scale in figure 4(d). Crosscheck of the computation quality can be seen, e.g. from the conduction-band minimum (CBM) (point $\mathrm{K}$ in figure $4(\mathrm{~b})$ ) and the valenceband maximum (VBM) (points $\Gamma$ ), which were found to be consistent with previous results [43]. It is noteworthy that the present band structure and gap calculations give very good agreement with experimental values. Other calculations, such as the generalized gradient approximation in the Perdue-Burke-Ernzerhof (PBE) functional form [44], may 
underestimate bandgap energies due to compromises between computational efficiencies and precisions.

The observed emission spectra at $\sim 1.9 \mathrm{eV}$ are attributed to transitions of excited electrons in the CBM to holes migrated just below the VBM. As shown in figure 4(c), the calculated energy difference between the peaks of DOS next to the Fermi level and DOS next to the VBM is $1.96 \mathrm{eV}$. This energy is in accordance with the observed PL energy. In the total DOS next to the Fermi level, the main peak is associated with a smaller one at a lower energy of $0.14 \mathrm{eV}$. The DOS next to the CBM is a singlet. Transitions of the electrons from these unoccupied to occupied states resulted in the PL features as observed in figure 2. In contrast to literature data, however, the VUV excited PL (figure 2) is stable at low temperature only, and is completely quenched at $100 \mathrm{~K}$. In addition it was not detectable at room temperature. We can explain these facts as follows. The present few-layered $\mathrm{MoS}_{2}$ is a semiconducting material having an indirect bandgap energy of $1.82 \mathrm{eV}$. Exciting this material by relatively high energy VUV photons we promote an electron in the CB with energy significantly higher than the CBM. After some relaxation, the electron reaches the bands next to the CBM. Meanwhile, the vacancies pop up in bands next to the VBM. Subsequently, the recombination between the electron and the hole results into the PL signal.

In the excitation case, the experimental absorption peak positions and relative intensities also agree well with the theoretical spectra. Detailed analysis of PDOS in figure 4(d) reveals that there are several substantial peaks around the Fermi level between -8 and $+8 \mathrm{eV}$. Such a big unoccupied DOS volume allows electrons from the VB to be prompted and kept therein, in accordance with previous explanations. The PDOS in figure 4(d) clarifies the origins of the observed peaks in figure 3 . The dominating spectral component originates from the electron transitions of the p- and d-DOS in the VB to the d- and p-DOS in the CB. Staying at $\sim 4 \mathrm{eV}$ below the Fermi level, both p-DOS and d-DOS have the most abundant densities. In addition, the d-DOS and p-DOS in CB peak at $\sim 3 \mathrm{eV}$ above the Fermi level. Similar energy intervals are found between the large VB DOS at $-2.6 \mathrm{eV}$, and CB DOS at $+4.5 \mathrm{eV}$ with respect to the Fermi level. The combined peaking densities of these occupied VBs and unoccupied CBs contribute to the observed strongest excitation peak at $7 \mathrm{eV}$. The present excitation scheme is different from the laser (e.g. $532 \mathrm{~nm}, 632 \mathrm{~nm}$ ) induced one where the electrons next to VBM are excited to the vacancies in the CB [17, 45]. Indeed, the experimental excitation spectrum may also bear other transition schemes due to the low-dimensionality of the material. Excitations were found to be strong in the theoretical results at $5.5 \mathrm{eV}$, but not visible in the experimental curve. Such peculiarities of the PL excitation spectrum may be correlated to the nano-scale. For instance, it can be supposed that low dimensionality strongly influences the oscillator strength of the transitions in $2 \mathrm{D} \mathrm{MoS}_{2}$. This leads to the appearance of the structure in the excitation spectra shown in figure 3. In other words, the measured PL excitation spectrum demonstrates the dependence of the transitions oscillator strength of few-layered $\mathrm{MoS}_{2}$ on low dimensionality in the VUV spectral range. Similar conclusions were reached in [33] in order to explain the dependence of the Si PL excitation spectra on the nanocrystal size. In the present theoretical calculations, however, such oscillator strength changes were not taken into consideration.

\section{Conclusions}

In this paper we have reported on the finding of a novel excitation peak in the VUV region of the few-layered $\mathrm{MoS}_{2}$ photoluminescence spectrum induced by energy tunable synchrotron radiation. An intensive PL excitation peaking at $\sim 7 \mathrm{eV}$ or $177 \mathrm{~nm}$ at low temperature was found beyond excitation energies supplied by conventional radiation sources such as lasers. From the temperature dependent PL spectra and first-principles calculations, the origin of the excitation peak was attributed to the intrinsic electronic structures of the low dimensional $\mathrm{MoS}_{2}$. Finally, considering the excitonic peak energy and the similarity of the present PL spectrum with the one obtained for Si nanocrystal, we suggest that few-layered $\mathrm{MoS}_{2}$ could be a transformer of UV/VUV radiation into visible light and may find practical applications as those reported in $[46,47]$ for multilayered van der Waals heterostructures.

\section{Acknowledgments}

We acknowledge the financial support from Oulu University Strategic Funding, Research Council of Natural Sciences of the Academy of Finland, the Swiss National Science Foundation, and the National Natural Science Foundation of China (No 51205001, No 11204079). The research leading to these results has received funding from the European Community's Seventh Framework Programme (FP7/2007-2013) CALIPSO under Grant Agreement No 312284. The authors thank also the crew of the MAX-IV laboratory for their support during the beamtime operation.

\section{References}

[1] Novoselov K S, Geim A K, Morozov S V, Jiang D, Zhang Y, Dubonos S V and Grigorieva I V and Firsov A A 2004 Science 306666

[2] Novoselov K S, Geim A K, Morozov S V, Jiang D, Katsnelson M I, Grigorieva I V, Dubonos S V and Firsov A A 2005 Nature 438197

[3] Geim A K 2009 Science 3241530

[4] Geim A K and Grigorieva I V 2013 Nature 499419

[5] Cohen S R, Rapoport L, Ponomarev E A, Cohen H, Tsirlina T and Tenne R 1998 Thin Solid Films 324190

[6] Furimsky E 1980 Catal. Rev.-Sci. Eng. 22371

[7] Hinnemann B, Moses P G, Bonde J, Jørgensen K P, Nielsen J H, Horch S, Chorkendorff I and Nørskov J K 2005 J. Am. Chem. Soc. 1275308

[8] Kim S et al 2012 Nat. Commun. 31011

[9] Lembke D, Bertolazzi S and Kis A 2015 Acc. Chem. Res. 48100

[10] Lopez-Sanchez O, Lembke D, Kayci M, Radenovic A and Kis A 2013 Nat. Nanotechnol. 8497

[11] Kibsgaard J, Chen Z, Reinecke B N and Jaramillo T F 2012 Nat. Mater. 11963 
[12] Wu S, Huang C, Aivazian G, Ross S J, Cobden D H and Xu X 2013 ACS Nano 72768

[13] Lee Y-H et al 2012 Adv. Mater. 242320

[14] Shi H et al 2013 ACS Nano 71072

[15] Espinosa F M, Ryu Y K, Marinov K, Dumcenco D, Kis A and Garcia R 2015 Appl. Phys. Lett. 106103503

[16] Splendiani A, Sun L, Zhang Y, Li T, Kim J, Chim C-Y, Galli G and Wang F 2010 Nano Lett. 101271

[17] Mak K F, Lee C, Hone J, Shan J and Heinz T F 2010 Phys. Rev. Lett. 105136805

[18] Tongay S, Zhou J, Ataca C, Lo K, Matthews T S, Li J, Grossman J C and Wu J 2012 Nano Lett. 125576

[19] Korn T, Heydrich S, Hirmer M, Schmutzler J and Schüller C 2011 Appl. Phys. Lett. 99102109

[20] Li J, Zhong Y L and Zhang D 2015 J. Phys.: Condens. Matter 27315301

[21] Yadgarov L et al 2014 ACS Nano 83575

[22] Cao W, Pankratov V, Huttula M, Shi X, Saukko S, Huang Z and Zhang M 2015 Mater. Chem. Phys. 15889

[23] Mahatha S K, Patel K D and Menon K S R 2012 J. Phys.: Condens. Matter 24475504

[24] Li D, Bancroft G M, Kasrai M, Fleet M E, Feng X H and Tan K H 1995 Phys. Chem. Miner. 22123

[25] Bunău O and Calandra M 2013 Phys. Rev. B 87205105

[26] Liu K-K et al 2012 Nano Lett. 121538

[27] Pankratov V, Kirm M and von Seggern H 2005 J. Lumin. 113143

[28] Pankratov V et al 2013 Radiat. Meas. 5613

[29] Pankratov V, Popov A I, Shirmane L, Kotlov A and Feldmann C 2011 J. Appl. Phys. 110053522
[30] Pankratov V, Popov A I, Kotlov A and Feldmann C 2011 Opt. Mater. 331102

[31] Kalinko A, Kotlov A, Kuzmin A, Pankratov V, Popov A I and Shirmane L 2011 Cent. Eur. J. Phys. 9432

[32] Pankratov V, Grigorjeva L, Chernov S, Chudoba T and Lojkowski W 2008 IEEE Trans. Nucl. Sci. 551509

[33] Pankratov V, Osinniy V, Kotlov A, Nylandsted Larsen A and Bech Nielsen B 2011 Phys. Rev. B 83045308

[34] Balasubramanian T et al 2010 AIP Conf. Proc. 1234661

[35] Urpelainen S et al 2010 AIP Conf. Proc. 1234411

[36] Zhao M, Dong W and Wang A 2013 Sci. Rep. 33532

[37] Huang H, Duan W and Liu Z 2013 New J. Phys. 15023004

[38] Bu H, Zhao M, Zhang H, Wang X, Xi Y and Wang Z 2012 J. Phys. Chem. A 1163934

[39] Ortmann F, Bechstedt F and Schmidt W G 2006 Phys. Rev. B 73205101

[40] Eda G, Yamaguchi H, Voiry D, Fujita T, Chen M and Chhowalla M 2011 Nano Lett. 115111

[41] Chow P K, Jacobs-Gedrim R B, Gao J, Lu T-M, Terrones H and Koratkar N 2015 ACS Nano 91520

[42] Li X et al 2015 Nanoscale 78398

[43] Cheiwchanchamnangij T and Lambrecht Walter R L 2012 Phys. Rev. B 85205302

[44] Perdew J P, Burke K and Ernzerhof M 1996 Phys. Rev. Lett. 77 3865-8

[45] Zeng H, Dai J, Yao W, Xiao D and Cui X 2012 Nat. Nanotechnol. 7490

[46] Withers F et al 2015 Nat. Mater. 14301

[47] Pospishil A 2014 Nat. Nanotechnol. 9257 